\title{
DEVELOPMENTS IN DIGITAL PRINT STANDARDIZATION
}

\author{
Christos Trochoutsos 2, 3 (1D), Anastasios Politis 1,2,3 (10) \\ ${ }^{1}$ University of West Attica, Graphic Arts Technology, Egaleo, Greece \\ ${ }^{2}$ Hellenic Open University, School of Applied Arts, Patras, Greece \\ ${ }^{3}$ HELGRAMED - The Hellenic Union of Graphic Arts \& Media Technology Engineers, \\ Athens, Greece
}

\begin{abstract}
Digital Printing has been established as one of the most rapidly evolving printing processes since its first introduction in 1982. In the years that followed, digital printing became the one significant new technology for print media production. Digital printing is continuously changing the print media landscape. Although, DP creates structural changes in production workflow and processes, it lacks in terms of print standardization, compared to offset printing for example, where consistent aim values and guidelines apply by means of ISO 12647-2. This drawback basically depends on two factors, which are interrelated. Firstly, there are many different technologies that are used in digital printing, and, each of them shows substantial difference in printing technology, substrates, data preparation, process control and image quality requirements. Secondly, compared to conventional printing, some digital printing technologies are still developing. After all, digital printing is versatile and variable in every way and cannot be standardized under a single standard.

A research on the digital printing technologies, processes and workflows is needed, to determine if a print specifications and quality controls (among them color management), can be applied in Digital Printing, and if possible, to which segment. Since color is very important to printing, especially in packaging and marketing applications, the print evolution demands for matching colors across technologies, substrates, materials and colorants. This paper intends to reveal the present status regarding Digital Printing Standardization. The question posed is whether standards can be applied and in which segments of digital printing either as technology or print sector (commercial decoration, packaging).

Within the paper, an analysis of the current industrial typical guidelines ranging from data creation all the way to printing will be made. Guidelines that are determined either by the manufactures of the digital printing machines, or by Institutes, such as FOGRA are reviewed for output process control and colour fidelity. As such, this paper can be regarded as a first attempt to preview the basis where standardization for digital printing processes can be developed.
\end{abstract}

Key words: Digital printing, Standardization, developments, technologies

\section{INTRODUCTION}

\subsection{Introduction}

Digital Printing (DP) has been established as a principal printing process since its first commercial introduction in the 90s. In the years that followed, digital printing became a significant new technology for print media production. Since then, Digital Printing has been developed rapidly and has brought about significant changes not only in printing itself but also in production workflow and in the total landscape of media market.

The establishment and the introduction of DP as well as its commercial application have led to predictions that this technology will eliminate traditional printing processes. Some older remember the title of an exhibition at a DRUPA (probably DRUPA 1982): "Good Bye Gutenberg". This "apostrophe" has been related with the digitalization of the production processes in the prepress field, the Internet and digital publishing, as well as with the introduction of digital printing.

\subsection{History of Digital Printing}

The history of digital printing is relatively short compared to printing as a whole, which dates back to 1439, when German businessman Johannes Gutenberg created a press that started the mass production of books. The first digital printing presses came onto the market in the early 1990s.

In 1993 the world's first digital colour printing press was launched called Indigo. The name of the printing press series came from a company formed by Benny Landa in 1977 to develop the world's fastest 
photocopier. Landa later discovered that the ink developed for the photocopier, called Electrolnk, could also be used in printers.

The first introduction of the Indigo printing machine, triggered a transformation in the printing world - all of a sudden, customers and print buyers were able to choose short-run, personalized, "high quality" print straight from prepress and the "desktop publishing" systems.

Print media industry expert Chris Baker, who worked as vice-president at HP and Indigo for five years, predicts that this growth will continue into the future: "Digital print will be everywhere in the future. Digital printing will not just be used for commercial printing; it will be used for publishing and packaging. I believe that digital presses will be developed to go after the packaging market - the personalization of packaging will be huge in the future. Digital presses will also become faster and be designed to handle more types of printing," (printed.com, 2020)

\subsection{Facts \& benefits of Digital Printing}

The growth of digital printing can be attributed by the many benefits claimed that offers to customers:

- Green: This method of printing can boost the green credentials of any company. Unlike conventional printing, there are no pre-press stages between the digital document file and the final print, so there is no need for film plates or photo chemicals. The process can be very environmentally friendly when water based inks are used and no powders or coatings are applied. It is sometimes cost prohibitive to go completely green but there are ways to keep costs down without harming the environment more than absolutely necessary.

- $\quad$ Speed: Digital printing offers a quicker response time due to its minimal press setup. It simplifies the printing process, traditional plates and film are redundant, there is no press make-ready needed, no plate mounting, no registration adjustments and no ink keys. There are less steps and people involved in the printing process, and as result the final product can be delivered quicker.

- Cost effective: Enabling companies to make financial savings is another benefit offered by digital printing. Traditional printing services have always had quotas or minimum orders required when you used their services. However, because of the flexibility of the printing press, digital printing companies do not have these sorts of boundaries, proving the freedom for the businesses and individuals to save and get the exact amount that they need.

- Short runs: Digital printing is the ideal method of producing short- to medium-runs in more effective ways than traditional print. Digital data is easily stored and updated; therefore changes are easily made either prior to printing or in the following batch. Digital printing allows more effective print management: there's no need for bulk stock and no need to dump out of date stock. Some machines that use digital technology can not only print out the materials but also finish the final product at the same time. (inline post-press)

- Print Enables Smarter Marketing: Variable data printing gives direct marketing a highly effective way to talk to customers, allowing companies to tailor their message to their audience. Delivering the right message to the right people at the right time. QR codes have also linked printed materials to the digital realm. Digital printing facts include information about variable data and variable imaging. Because computers control the printing process it is much easier to change the content of the item while it is being printed. This adds a new level of customization and personalization that can greatly improve your finished product appeal. (PEARL, 2013)

\section{GUIDELINES AND SPECIFICATIONS FOR COLOR PRINTING}

\subsection{Color image quality in printing}

The publishing industry is constantly changing. New disruptive digital printing techniques enter the market, standards continue to evolve or to be revised; electronic media allows print service providers to become communication providers. That implies more complex processes and related interactions.

Color image quality is a very important deciding factor for buyers of color imaging devices. It is therefore of utmost importance for manufacturers of imaging equipment such as digital presses, to pay special attention to this factor. After several years of market hesitation, digital presses have now become common (most of them). In today's printing market, where flexibility, variable content, shorter lead times, and on demand publishing, are being demanded, digital presses represent an attractive 
supplement to conventional offset presses. It is important for customers of digital press equipment to be able to take into account the quality of the equipment, typically to be able to make a trade-off between price and quality. The total quality of a device is, however, a very complex entity, involving technical aspects such as expected lifespan, printing speed, accepted media, as well as customer relation aspects such as service agreements. (FOGRA, 2018)

There are indeed many factors that contribute to the quality of an image, such as spatial resolution, color depth, sharpness, naturalness, colorfulness, and visual artifacts (banding, streaking, grain, blocking, moire, etc.). There exists an ongoing effort to standardize the definitions of these and other image quality factors, as well as their assessment methodology.

Potential uses of quantifiable data on color image quality for manufacturers include the following:

- Tradeoff analysis of speed and implementation cost versus color image quality in image processing algorithm development

- Benchmarking of imaging systems and algorithms to other vendor's products

- Documentation of color image quality improvements resulting from efforts spent on optimization of technology parameters

For customers, it would obviously be advantageous to have access to reliable and objective information about the image quality that devices can provide when considering several alternatives for purchasing. Every print shop is committed to a high level of quality. A basic prerequisite for this is to use meaningful rules, hence a standard. In standardization a distinction is often made between the specification of the final aim and the needed (process) steps to achieve that aim. A good example for the latter one is the PSO - Process Standard Offset, which has been successfully in place for years. (Hardeberg and Skarsbo, 2002)

\subsection{Color management}

Two main components are needed to successful color management, no matter in offset printing or in digital printing. These are the technology part (meaning to have the appropriate hardware and software) and the proper education (understanding color management theory and the workflow knowledge).

Hardware is complicated, as it insists of different digital printing presses (and there are literally hundreds of models), different ink sets, different ink types, different substrates and so on.

Color measuring devices are used to measure the resulting colors of ink mixtures on the substrate. Standard devices are designed to measure on flat and even surfaces. Substrate properties can interfere with the measurement. Measuring colors correctly on those surfaces requires measurement equipment designed for that purpose. More information about measuring in different conditions can be found in section 2.4. (Gerrit, 2017)

Some of the major construction companies for digital presses have developed integrated tools to simplify and automate the colour management process. These tools ensure that the colour accuracy remains consistent and uniform across presses and sites and over time, without laborious manual calibration.

These tools usually consist of an Inline Spectrophotometer scans a colour chart to describe the individual colour space of a specific substrate (the "Media Fingerprint"). The DFE (Digital Front End) then automatically generates a dedicated ICC profile, ensuring accurate colour matching of that specific substrate against a given FOGRA, GRACOL etc. tool based on standard such as ISO 12647-2.

Accurate matching to these industry standards also enables seamless emulation of output from other print technologies (such as offset). A three-dimensional LUT is then used to regularly calibrate the press, automatically compensating for any variations in press conditions to return it to the baseline established by the Media Fingerprint, ensuring that colour output always remains consistent. On web-fed presses this process happens at a pre-determined interval, whereas on sheet-fed presses the operator is free to determine the frequency of calibrations. Media Fingerprints can be saved and shared between presses and sites, and over time, to ensure a consistent output regardless of where or when the file is printed.

\subsection{Why digital printing is hard to standardize}

Since there are many different printing technologies, processes and workflows it is under research to determine if a color management process can be applied in digital printing, and if possible, to which applications of digital print. The physics of the lithographic process doesn't vary from one model or machine to another. But, all digital press manufacturers have unique, patented processes. 
The very term "digital" is misleading, because it refers to many disparate processes. Digital is commonly taken to mean toner/laser technology, but in the printing world, it may just as readily mean inkjet. Laser and inkjet are about as different as anything can be.

In the digital process, a crucial consideration for color quality is gamut-the range of color that a press can reproduce. The wider the gamut, the more colors can be printed. But gamuts of equal size are not always equal. One device may be weak in reds, another in blues. Sometimes, same size gamut can produce very different results.

Usually, digital presses have a wider color gamut than the offset process. Because the offset gamut is still the standard in many people's minds, this is often not understandable and is a more confusing fact. Unlike offset, each digital press model has a difference color gamut. All are different from offset. And it's even more complicated when considering also the spot colors reproduction and not just the conventional CMYK colors. There is also a belief that because of the massive range of applications, which is one of the key benefits of digital printing, this also makes it hard to standardize.

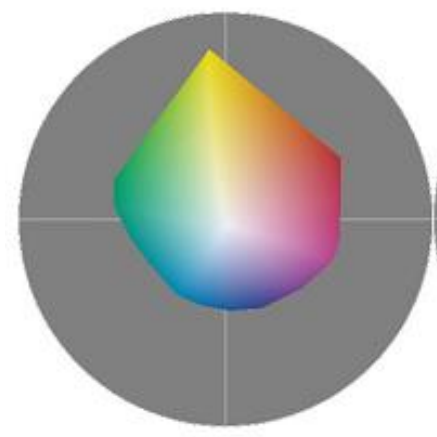

(a) Océ CPS700

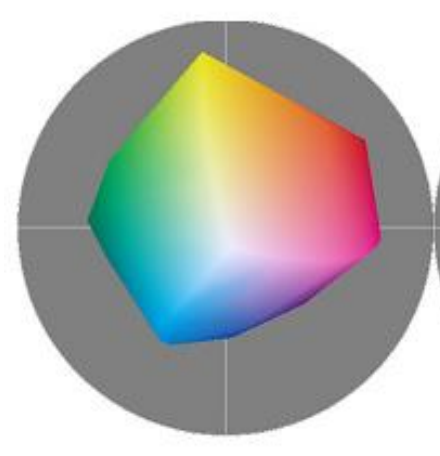

(c) Canon CLC5000

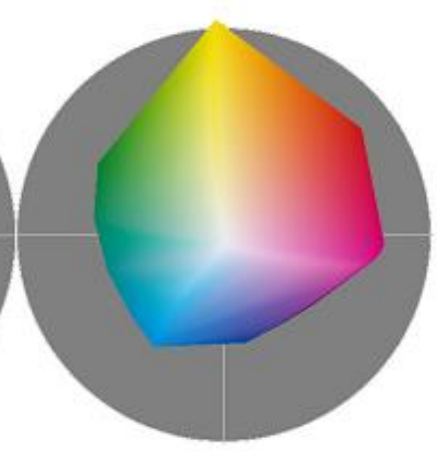

(b) Xerox DC2060

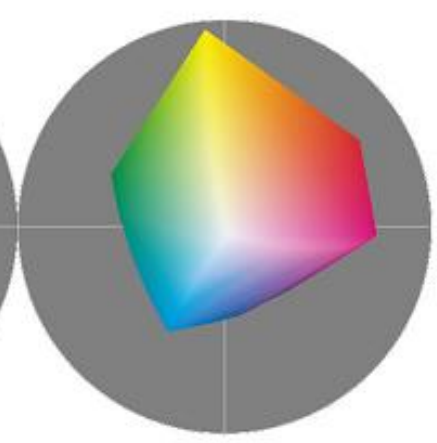

(d) NexPress 2100

Figure 1: Different digital presses, different color gamuts

\subsection{ISO 12647-2: Process Standard Offset}

The Process Standard Offset (PSO) was developed by Fogra in co-operation with the German Printing And Media Industries Federation. It is the description of an industrially orientated and standardized procedure for the creation of print products. The PSO is in conformance with the international standardization series ISO 12647 and therefore internationally recognized.

By using PSO the quality of the production of a complete print product can be guaranteed, from data creation to the finished printing product. The PSO describes adequate testing devices and control methods by which the production process can be supervised, guided and proved. This includes measuring devices with spectral and densitometric settings, as well as suitable testing elements (for example test stripes). Furthermore, the PSO sets nominal values and tolerances for the print production that, relating to modern production materials, represents what is sensible and feasible. (printed.com, 2020)

The goal is to ensure that the production process is as efficient as possible and at the same time to ensure that interim and final results show a predictable quality of colour. In the printing 
industry very rarely data is printed where it was created. Customers often hand jobs to different print and media specialists. Printing plants collaborate in order to concentrate themselves on their core competences. This trend will continue and will extend across borders as well. The PSO and ISO 12647 are in constant development in order to benefit the user. This way, quality becomes measurable, verifiable and reproducible. ISO 12647-2 is part of the ISO 12647, which contains the following:

- $\quad$ ISO 12647-1 - Part 1 Parameters and measurement methods

- ISO 12647-2 - Part 2 Standardized offset printing

- $\quad$ ISO 12647-3 - Part 3 Standardized press printing

- $\quad$ ISO 12647-4 - Part 4 Standardized gravure printing

- $\quad$ ISO 12647-5 - Part 5 Standardized screen printing

- $\quad$ ISO 12647-6 - Part 6 Standardized flexo-printing

- $\quad$ ISO 12647-7 - Part 7 Standardized digital printing

Unfortunately, part7 is not about digital printing products, but for proof prints, that are printed with digital printers. (gmg, n.d.)

Process standard offset is a living process, so as the paper technology and other factors that affect printing changes, the standards may also change. For example, latest ISO 12647/2-2013 printing conditions and Fogra 51 and 52 it's the revised ISO standard printing conditions for sheet and web fed heatset-offset litho. This revision was published at the end of 2013 and it replaces the 2004 version. The main changes to the revised standard and the new data sets and profiles being developed by Fogra for coated and uncoated papers. Changes are also made due to the implications of the new spectrophotometer specifications, M1, in relation to the new 2013 version and the Fogra data sets.

- $\quad$ First, papers have been revised to better reflect the papers in current use with their high OBA (Optical Brightening Agents), so there are now 4 coated and 4 uncoated paper types in this new version, three more than the old 2004 version. These are now called 'Print Substrates' (PS), replacing the 'Paper Types' (PT) is the 2004 version.

- Colour differences are still measured and evaluated using De76, however De2000 tolerance values are also given.

- $\quad$ The new specifications for CIE Lab figures and TVI curves will result in new colour data sets and ICC profiles being needed. At present Fogra are working on a coated profile for PS1, based on a new dataset, Fogra 51 and an uncoated profile for PS5, also based on a new dataset, Fogra 52.

- This new version of $12647 / 2$ is much more than just changing to new ICC profiles and new measurement figures. This change also may need new lighting, new spectrophotometers and changing digital proofing papers to have OBA/UV content.

One of main factors as referred to above for this new version of $12647 / 2$ is to reflect the use of OBAs in papers. The viewing and lighting conditions standard, ISO 3664 was revised in 2009 in order to support the extra UV emissions caused by the OBA content. So, this is now within the standard and the latest D50 tubes will meet this requirement.

However, to measure accurately printed sheets a new spectrophotometer measurement standard is needed, M1. The 2004 standard uses M0 measurements, which do not correctly record the UV content of the paper, so can affect the accuracy of the ink colour readings.

The M1 spectrophotometer measures and allows for the UV content caused by the OBAs. For example, an $\mathrm{M} 0$ reading giving $\mathrm{b}$ at -4 , on reading using $\mathrm{M} 1$ would result in a more accurate $\mathrm{b}-6$.

Older spectrophotometers will not be able to read in the M1 mode; so, some updating of instruments is inevitable, both to off-line handhelds and on-press online devises.

Current 'approved' inkjet proofing papers are often OBA free. Proofing papers for the new profiles and M1 spectrophotometer readings will need to match the OBAs in the chosen paper type as closely as possible. (Sherfield, 2014)

\subsection{PSO and Digital Printing}

After the success of the Standardization for Offset, the production also needs Digital Print Standardization for matching colors across technologies, substrates, materials. Process control needs to cover all print specific settings and the corresponding visual and instrumental measures, in order to establish a repeatable and stable printing condition. The process control measure therefore strongly depends on the 
printing technology and media used. Although process control is considered to be the responsibility of the print service provider some basic principles are important.

In the majority of such applications the requirement could be termed as "matching offset". In light of this it stands to reason that where possible it would be good to make use of the established means to control offset printing processes. This would include the usage of the same control wedges, measurement devices [in particular densitometers to measure the tone response] and, last but not least, the evaluation and the comparison of digital prints by means of press acceptance tests established for offset printing.

Press acceptance tests usually cover the solid coloration, the tone value increase [formerly known as dot gain] as well as grey reproduction. Those figures are compared against aim values derived from state of the art characterization data sets such as FOGRA39. Whilst ISO 12647-2 and hence PSO make provisions for a binary [conforming or not-conforming print] evaluation, manufactures of such solutions also offer a single value index also known as scoring value. Such scores typically range from 0\% to 100\% or from 1 star to 5 stars. The problem with these scores is that there is no agreed basis for their calculation so any score is somewhat proprietary and can't be compared among different vendors.

But Process Standard Offset can't be applied in Digital Printing it the same way. Contrary to digital printing the binary [Yes/No] evaluation of offset prints represents the results of many years' research and practical implementations. Still, some problematic aspects describe why the "offset concept" can't be transferred without some modification to the digital domain. That's the reason, why Fogra's working group created a new digital printing standard ISO 15311. Three of these main aspects are described below:

1. Inadequate definition of the pertinent scope. The usage of PSO for digital printing needs to be critically reviewed as there is no description as to what technologies and substrates it should be applied to. The term "digital printing" as a description for a given printing technology or imaging process is quite unsuitable. While the use of PSO for digital printing might be considered intuitive for toner based systems it is more than questionable for large format banner printing using UV-curing inkjet or textile printing using thermal processes.

2. Lack of scientific knowledge and practical experience. The various digital printing technologies are compared to conventional printing processes at a relatively early stage of their development and so that standardization activities and the necessary research is still scarce. This means that the implementation of procedures and methods which are successfully used in offset printing are not necessarily transferable to every kind of digital printing. Rather, such a "transfer" must be tailored and optimized for each use case, considering strengths and weaknesses of the pertinent imaging processes. Examples include the typical inkjet banding or a characteristic graininess as is found in some inkjet printing systems induced by coalescence. These digitalprinting-specific problems might severely affect the final image quality but are not included in the "offset evaluation method toolbox". An audit or a compliance check in accordance with PSO ignores this property completely.

3. Missing Link to underlying process parameters. The primary aim of densitometry is to monitor the amount of colorant per area on a print. In order to measure the tone reproduction [the tonal response $[\mathrm{CIEY}]$ of the primary colours from $0 \%$ to $100 \%$ ] the graphic arts industry uses the tone value, better known to some as apparent dot area. The solid densities and the tone value increase helped conventional process control reliably by indicating [with high sensitivity] press problems and monitoring the relative changes in tone reproduction of an image as it moved through the various stages of data preparation to a printing plate and eventually to the printed image. The underlying concept in ISO 12647-2 is that once the correct process colour solids and two-colour solid overprints are achieved, a satisfactory overall result can be reached by simply adjusting the tone value curve to match the specified tone value curve defined for the pertinent printing condition. In general, this assumption can't be made for digital printing although it is possible to measure solid density and tone value increase. This doesn't allow for any direct link to the underlying process parameters since they can't be easily identified. They are dependent on the imaging process and the interaction of the colorants with substrates used. For example, there is no doubling in inkjet printing. A possible evaluation of established doubling-slur patches thus leads to results that are hard to interpret by the user or are simply meaningless. Further it should be noted that densitometers mostly use a cross polarizer to reduce the effect of first surface reflections which reduce the measured differences between wet and dry prints. This may not be suitable for many digital printing technologies since it differs from colorimetrical readings 
which are made without polarization filters. Note that the machine's internal use of density measurements for dedicated control processes, for example the control of the toner transfer to the photoconductor, is not considered as being inappropriate [it actually might be a very good solution]

\subsection{Digital Print Standardization - ISO 15311}

The basic prerequisite for printing predictable print quality is to use meaningful rules, hence a standard. In standardization a distinction is often made between the specification of the final aim and the needed (process) steps to achieve that aim. For digital printing the PSD - Process Standard Digital provides industrial typical guidelines ranging from data creation all the way to printing and is provided by Fogra. The ISO title is: Graphic Technology - Requirements for printed matter for commercial and industrial production. However, there is some discussion within ISO TC130 WG3 if the standard should be restricted to digital printing or not (since it can be applied also to conventionally printed products).

Contrary to ISO 12647-x, ISO 15311 is a multipart standard based on representative use cases (rather than printing technologies). Substantial contribution has been made in the Fogra digital printing working group (DPWG). The current structure looks as follows:

- $\quad$ ISO 15311-1 - Part 1 Parameters and measurement methods

- $\quad$ ISO 15311-2 - Part 2 Commercial production printing (almost published)

- $\quad$ ISO 15311-3 - Part 3 Large format printing (draft will be published as Fogra specification)

- $\quad$ ISO 15311-4 - Part 4 Additional parts based on use case (in discussion)

As far as the problematic aspects appear in digital printing in comparison to offset printing, Fogra proposes the following solutions:

1. The recently started multi-part standard ISO 15311 [Graphic Technology - Requirements for printed matter utilizing digital printing technologies for the commercial and industrial production] will provide a process-independent classification of the relevant use cases and applications and these will be accurately defined. Thus, for example, part 2 of ISO 15311 addresses commercial production printing while part 3 stipulates aim values and tolerances for large-format signage printing.

2. Fogra has applied for a research project that has started recently. It focuses on the development [and improvement] of objective methods for evaluating inhomogeneity and sharpness. First ideas were discussed during the last meeting of the DPWG [Fogra Digital Printing Working Group] and in the October 2010 ISO/TC130 WG3 meeting. In the short- and mid-term results from this research project can be expected that contribute to the vendorneutral, objective evaluation of the pertinent print image quality attributes.

3. Many manufacturers of digital printing systems are moving towards a colorimetric evaluation or inspection of their prints. This begins with the adjustment and calibration, via ICC profiling and ends with the colorimetric print quality evaluation. This development is already covered in the process independent definitions of contract proofs [ISO 12647-7] and the so-called "Validation Prints" [ISO/DIS 12647-8]. Both are fully defined without using any densitometric methods. The DPWG [Digital Printing Working Group] is working on a fullyfledged all colorimetry-based metrology including the respective statistical analysis [and uncertainty analysis] for process control and quality assurance (Kraushaar, 2010)

The Process Standard Digital has three main objectives:

- Output process control - Achieving a repeatable print output. Different output processes need to be checked against their known and constant behaviour.

- Colour fidelity. The second goal addresses a consistent colour communication by means of faithful image reproduction. Guide by the motto: "Printing the Expected" quality-oriented print service providers do first understand the needs and expectations of their clients and are second able to accurately reproduce that expectation. In that light the Process Standard extends the established way of colour reproduction namely the absolute reproduction ("Side-by-Side") by means of an all new media relative colour reproduction.

- PDF/-X compliant workflow. As in this paper, an analysis of the current industrial typical guidelines from data creation all the way to printing will be made, it's important that the entire workflow is subject to critical scrutiny as to its capacity for sustained achievement of consistent 
print quality and colour fidelity. So, Process Standard needs to provide guidelines for creating, preflighting and processing PDF based documents.

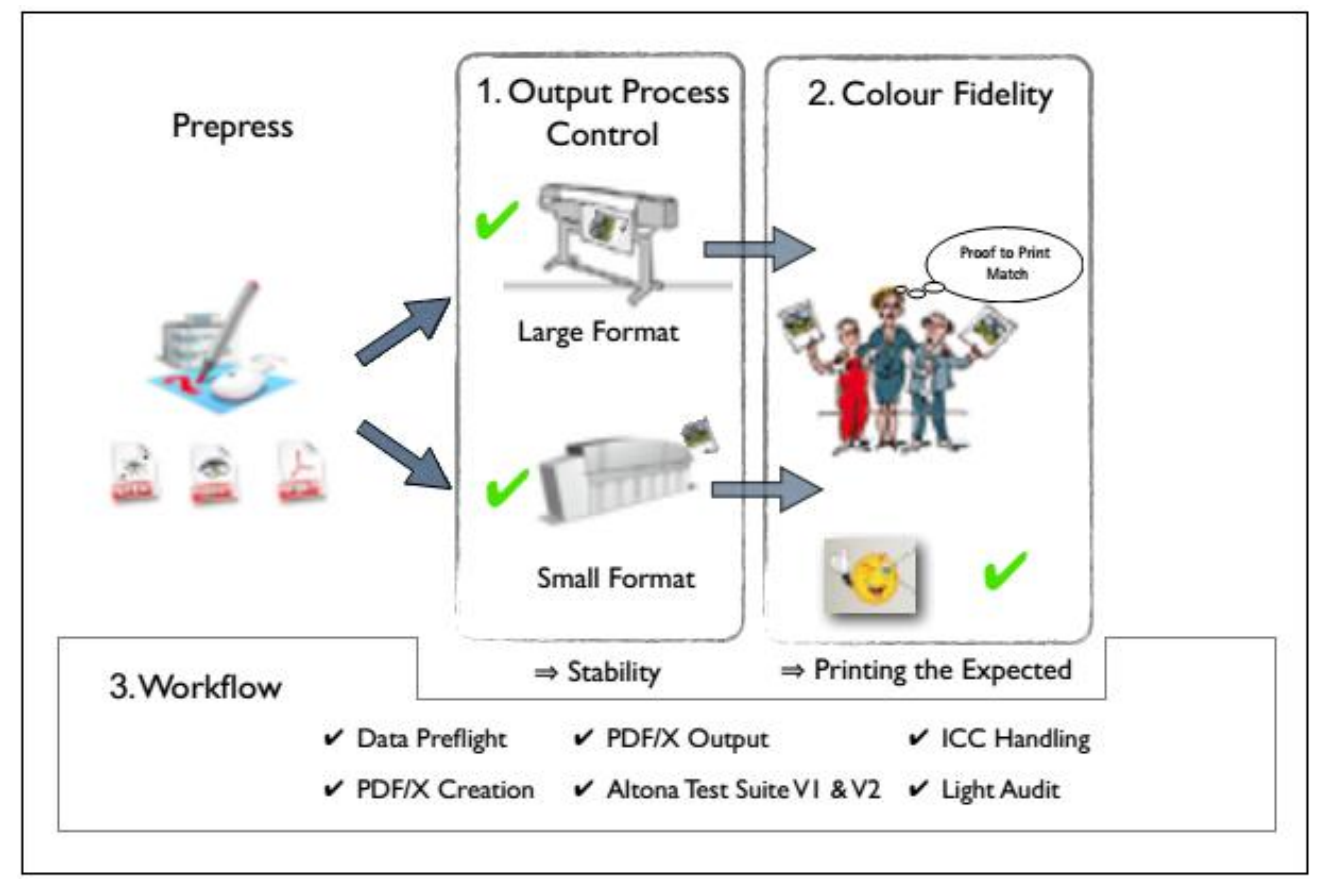

Figure 2: Fogra PSD-concept. Aiming for a consistent and predictable print quality.

Standardization does not mean that materials such as substrates, inks, toner or machinery must be limited. On the contrary, standardization aims toward a manageable facilitation of a material and process diversity in terms of rigors and consistent print quality. Only then is it possible to identify suitable "combinations", i. e. collection of a driving (RIP \& colour management), a substrate, ink or toner and a printing press. Although many factors are the same in almost every digital printing press (for example the E.F.I. Fiery is used in most cases for RIP), as mentioned before, the publishing industry is constantly changing. New disruptive digital printing techniques enter the market. From xerography, to electrophotography and the latest one: nanotechnology, which is still being developed. That implies more complex processes and related interactions. To meet these changes the standardization process is designed as a "living document".

\subsection{Factors that affect quality of Digital Printing}

The color gamut of a digital press (or any other imaging device) is the sum of all colors it can reproduce. Colors that are outside of this gamut cannot be reproduced. The color gamut, and in particular its size, is thus a quality factor. A press with a larger gamut than another is typically able to reproduce more saturated colors, which can be appreciated for many applications. The color gamut of a digital press depends on many factors such as substrate, toner/colorant, and halftoning algorithm. And unfortunately, every digital press has its own color gamut, as mentioned in section 2.3.

\subsubsection{Substrate}

Two main types are available: swellable and porous. In general, acid-free, buffered, and lignin-free paper bases are recommended for long-term storage.

Using swellable paper, its surface of this type of inkjet paper swells in the presence of the moisture in the water-based ink, allowing the colorants to penetrate the top layers. Swellable papers typically have three active layers: a protective top layer, a layer that fixes the ink droplets in place, and, below that, a layer that absorbs additional ink components. The paper base is sandwiched between two polyethylene layers and backed by an anti-curl coating and an antistatic layer. Premium swellable papers contain all of these layers, but less expensive ones may not. The polymer coating on swellable papers acts to maximize image 
brightness by keeping the colorants from spreading, and, to some extent, it protects the image from atmospheric pollutants. Images printed on coated paper may require a significant amount of drying time. On the other hand, the surface of porous paper is coated with very small, inert particles, which create numerous minute cavities in which the ink is deposited. These particles prevent the ink from spreading. Porous paper has a higher resistance to moisture and humidity. This paper requires minimal drying time, so the print can be handled immediately without fear of smudging. This type of print has no protective polymer layer; therefore, the colorants are susceptible to attack by atmospheric pollutants, such as ozone and oxides of sulfur and nitrogen, which can be present in the environment in fairly high concentrations. Porous paper is preferred when pigment inks are used.

That's why in ISO 12647-2 there are different standards regarding the different type of paper, and the same difference have to be considered on digital printing.

\subsubsection{Toner/Colorant}

Dye Diffusion thermal transfer Prints In this process, which is also referred to as dye sublimation, heat transfers colorant from a donor ribbon to the final print. Dye diffusion thermal transfer printers frequently apply a clear protective layer to the print during the transfer process to prevent the image from smearing when rubbed. This process is frequently used for snapshot-size photo printers or in photo kiosks, where customers can print images in a few minutes. The permanence of these images has not been widely studied, and there is very little information available.

The majority of photo-quality printers use inkjet technology, in which very small droplets of ink are deposited onto paper. Inkjet prints vary widely not only in the composition of the colorants and paper, but also in stability. Inkjet images may be composed of dyes like those used in traditional photographic prints, or of pigments, which are the colorants used in paints. In general, pigments tend to be more stable than dyes.

Inkjet prints can be made on either uncoated or coated paper, but only coated paper will provide photoquality prints. Plain, uncoated paper tends to absorb the ink, resulting in a blurred image and loss of color intensity. In high-quality paper, a coating prevents the ink from bleeding into the paper; this results in brighter, more saturated color and greater image detail. Coated papers may closely resemble traditional color print supports. (IPI, 2004)

In electrophotographic prints, the same process used to produce office photocopies is used to make the prints. In electrophotography, toner is transferred to the paper base and then fused in place. The paper is usually uncoated, and the images are reasonably stable, because they are composed of pigment particles that are fused to the paper with a durable polymer binder material. This technology is used less widely than inkjet for photo-quality printing (Fogra, 2018) Electrophotography is the most widespread nonimpact-printing technology that exists. Many companies, like Xerox and Canon use variants of the electrophotographic principle with electrostatic powder toner, based on an invention of Chester Carlson from 1939 (HP, 2012).

\section{TYPICAL GUIDELINES RANGING FROM DATA CREATION ALL THE WAY TO PRINTING}

Printing the expected is not easy. To standardize printing we need to have the same printing results and not just print the expected, but have the same output across different printing technologies, different substrates, production batches.

Problems might occur not only on the output procedure, but in many occasions in input file formats as well. PFD files are container files and elements can include bitmap graphics in different color modes (RGB, CMYK, LAB, ...), vector elements, spot colors, overprints, transparencies, etc.

Depending on the creation application the result can be overly complex which leads to longer processing time and mistakes during PDF processing. Missing color management information (embedded profiles and output intent) leaves room to interpretation and errors.

$\mathrm{PDF} / \mathrm{X}$ is the ideal data exchange format for the printing industry. The purpose of it is to facilitate graphic content exchange with specific requirements linked to production workflow needs. In the following table some typical tasks of the prepress side are provided to avoid faults. 
Table 1: Typical tasks together with the corresponding party being responsible for that specific job.

\begin{tabular}{|c|c|c|c|}
\hline Nr. & Task & Responsible & Note \\
\hline 1 & $\begin{array}{l}\text { Data creation - open } \\
\text { (application) files }\end{array}$ & $\begin{array}{l}\text { Print service provider } \\
\text { should inform the } \\
\text { data deliverer about } \\
\text { the status of the data } \\
\text { integrity. }\end{array}$ & $\begin{array}{l}\text { Providing a reliable visualization e.g. by } \\
\text { means of a hard- or softproof based on the } \\
\text { corrected (optimised) data is needed for } \\
\text { colour critical jobs. }\end{array}$ \\
\hline 2 & $\begin{array}{l}\text { Providing Print-ready } \\
\text { data - marked as } \\
\text { PDF/X. }\end{array}$ & Data deliverer & \\
\hline 3 & Preflight of data & Print service provider & \\
\hline 4 & $\begin{array}{l}\text { Agreement and } \\
\text { negotiation about } \\
\text { the output condition } \\
\text { (printing condition) }\end{array}$ & $\begin{array}{l}\text { Print service provider } \\
\text { together with the cli- } \\
\text { ent (print buyer) }\end{array}$ & $\begin{array}{l}\text { These include the definition of the quality } \\
\text { type for CMYK (and spot if present), the } \\
\text { intended viewing distance, the reference } \\
\text { characterization data set as well as the } \\
\text { colour reproduction (.Side-by-Side" or } \\
\text { media relative) }\end{array}$ \\
\hline 5 & $\begin{array}{l}\text { Printability of the } \\
\text { substrate }\end{array}$ & Print service provider & $\begin{array}{l}\text { Unless the manufacturer of the print- } \\
\text { ing system has "certified" the pertinent } \\
\text { substrate. }\end{array}$ \\
\hline 6 & $\begin{array}{l}\text { Process Conversion } \\
\text { when data is not pre- } \\
\text { pared for the intended } \\
\text { printing condition }\end{array}$ & Print service provider & $\begin{array}{l}\text { The print service provider is required to } \\
\text { inform the print buyer about potential } \\
\text { limitations preferably with a contract proof } \\
\text { based on the re-separated data. }\end{array}$ \\
\hline
\end{tabular}

During the printing process, even more tasks are needed to ensure the right output. A printing system is only as good as its maintenance status. A printer has to:

- Follow manufacturer periodically recommended operations

- Ensure that both printing system and substrates used are in appropriate environment conditions

- Identify and check material combination

- Choose the desired print mode(s)

- Understand specific parameters for printer (hardware) and workflow (software)

- $\quad$ For drying/curing printing systems, the temperatures applied during printing and post-printing stages are very important for both printability and runnability

- Select colour reference(s). The achievable colour gamut results from the substrate and ink interaction and performance

Most important one the measurement procedure. To control colour adjustments and calibrations are needed and in many occasions the printer must create a specific characterization and profiling (e.g. ICC) for the specific job. This is not needed in offset printing, since the ICC profiles that are being used are provided by ISO 12647. In digital printing the characterization data set for the selected printing combination must be created and then the printing condition becomes a fully characterized printing condition. In this stage a typical ICC Output Profile will be generated, but in some occasions a device link profile may be also used.

Lastly, to validate the printing output the stability of the printing system for a given printing condition needs to be checked, as well as the deviation from aim values of the printing condition to be simulated. This is done by using the control strip or chart, using the properly measuring device in proper conditions and check the pass/fail tolerances adapted from already established validation print tolerances.

Periodically calibration or re-calibration is recommended in case of a faulty evaluation. Monitoring and recording the pass/fail evaluation for tracking purposes is also recommended as it will show up trends useful to fix issues before they become problems. 


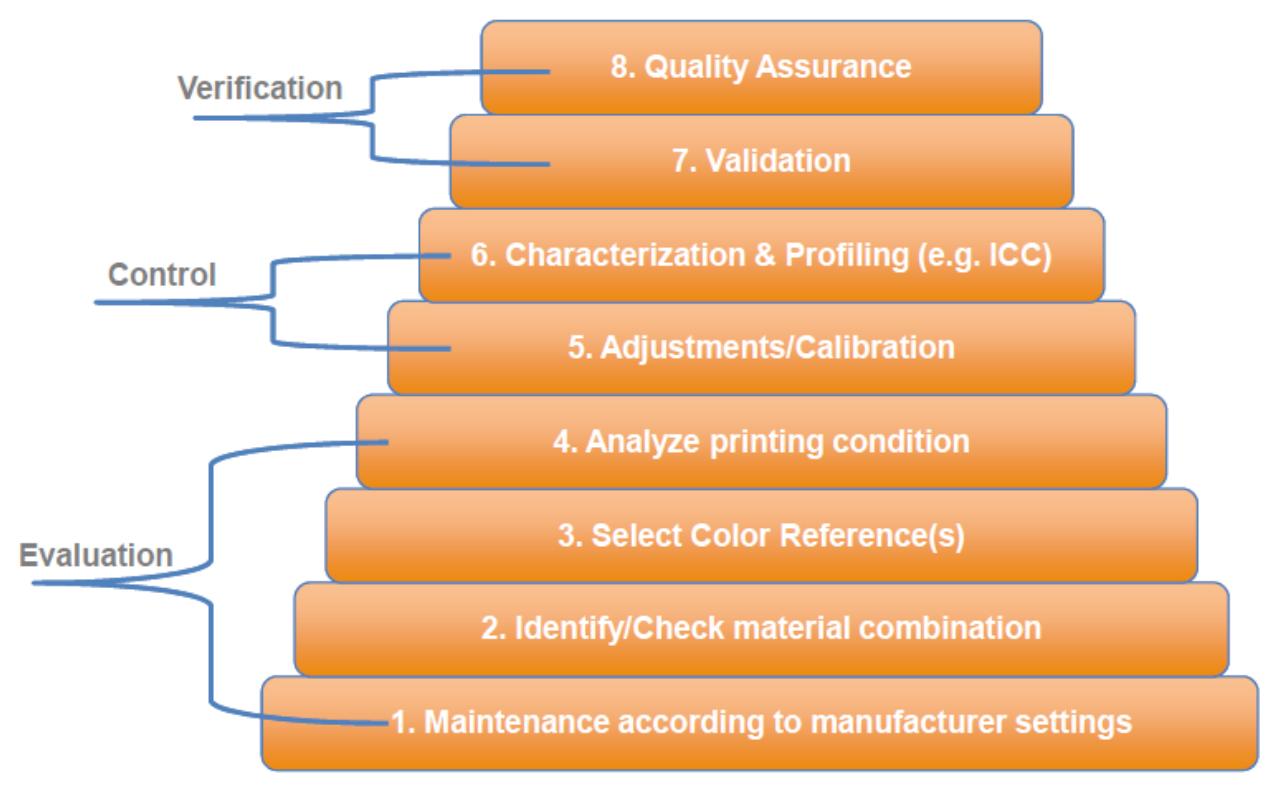

Figure 3: Process Control pyramid - 8 steps to success

\section{FURTHER RESEARCH}

Color image quality is of very high importance in a digital imaging device such as a digital press. For manufacturers and customers of such devices it is important to be able to quantify color image quality. Unfortunately, as of today there are no analytical techniques that can quantify color image quality in this context. Contrary to ISO 12647-x, ISO 15311 is a multipart standard based on representative use cases (rather than printing technologies). Despite this fact, some researches rely on experiments using ISO 126147-x. Some others rely on experiments involving real observers. We claim that the notion of color image quality is ultimately tied to the preferences of customers and end users. Because of this, a very useful tool to quantify color image quality is psychophysical experiments involving a panel of human observers. However, such experiments are relatively time consuming. Definitively, Yendrikhovskij (1999) hits the nail on the head when he states that "most studies on image quality employ subjective assessment with only one goal - to avoid it in the future." Therefore, results from ongoing research toward analytical models for color image quality is eagerly anticipated. An example of such research is the development of metrics for color differences between complex images. However, much more use cases must be analyzed in different digital presses, different substrates, different settings etc. to assure a standardize process in digital printing. And this is even more complicated, since digital printing consists of commercial printing, large format printing, printing in special materials and so on. And nobody knows the evolution of digital printing by using nano technology. Landa's presses are believed to print the same output no matter what (any size, any substrate and matching ISO 12647-2). Will this set a new standard for digital printing?

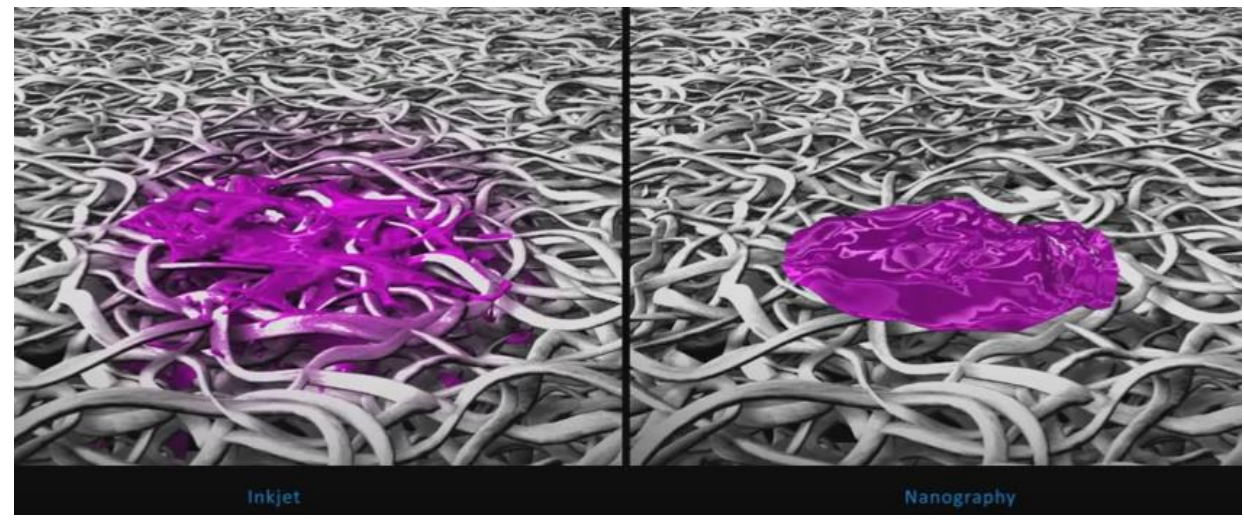

Figure 4: Landa's nano-pigment toner penetration to substrate in comparison with inkjet 


\section{CONCLUSIONS}

In summary, it can be stated that the underlying concept of ISO 12647-1/2/3 [including the recently started revision] is completely adequate for offset printing. In contrast, digital printing tailored to specific technologies is hard to imagine. Instead, a process-independent concept that addresses the individual applications with dedicated print quality measures seems more appropriate. The demand for a digital printing standard alongside methods for process control and quality assurance is evident. The use of established methods in offset printing, in particular by offset printers that also use digital printing systems might be quite useful - but it comes quickly to many limits that are outlined in this paper. Although it might be tempting to simply use the existing PSO system for digital printers, too, this has a poor technical basis and is, thus, likely to reduce the industry's confidence in certification altogether. Fogra is making research on Process Standardize Digital and there's still a lot of space for further research, as mentioned above. The various digital printing technologies are compared to conventional printing processes at a relatively early stage of their development and so that standardization activities and the necessary research is still scarce. This means that the implementation of procedures and methods which are successfully used in offset printing are not necessarily transferable to every kind of digital printing.

\section{REFERENCES}

[1] Fogra: "Process Standard Digital Handbook, Step by step toward printing the expected", (Fogra Research Institute for Media Technologies, Bayern, 2018.)

[2] Gerrit, A.: "Innovative Solution Committed to Color, Color Management for Industrial Printing Applications", URL: http://www.inprintshow.com/usa/conference/pdf/Gerrit-Andre.pdf (last request: 2020-09-28), 2017.

[3] gmg: "New ISO standards for proof prints", URL: https://www.gmgcolor.com/specials/new-isostandard/ (last request: 2020-09-28)

[4] Hardeberg, J. Y., Skarsbo, S.: “Comparing color image quality of four digital presses", URL: http://www.ansatt.hig.no/jonh/archive/ipgac02_iq.pdf (last request: 2020-09-28), 2002.

[5] HP: "HP Indigo Digital Offset Colour Technology", URL: http://directedonline.com/wpcontent/uploads/2014/08/HP-Indigo-Tech-F.pdf (last request: 2020-09-28), 2012.

[6] IPI: "A consumer Guide to traditional and digital print stability", URL: https://scrlc.org/data/consumerguide_traditional.pdf (last request: 2020-09-28), 2004.

[7] Kraushaar, A.: "Process Standard Offset print for Digital Printing?", Fogra extra 23, 2010.

[8] PEARL: "The digital future of print", URL: http://www.pearlprintdesign.com/the-digital-future-ofprint/ (last request: 2020-09-28), 2013.

[9] printed.com: "History of Digital Print", URL: https://www.printed.com/history-of-digital-print (last request: 2020-09-28), 2020.

[10] Sherfield, P.: "The new ISO 12647/2 -2013 standard printing conditions Fogra 51 and 52", URL: http://www.missinghorsecons.co.uk/wordpress/2014/06/the-new-iso-126472-2013-standardprinting-conditions-and-fogra-51-and-52/ (last request: 2020-09-28), 2014.

[11] Yendrikhovskij, S. N.: "Color reproduction and the naturalness constraint", PhD thesis, Eindhoven University of Technology, 1999.

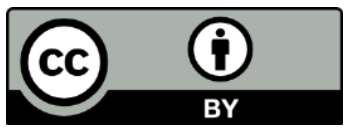

(C) 2020 Authors. Published by the University of Novi Sad, Faculty of Technical Sciences, Department of Graphic Engineering and Design. This article is an open access article distributed under the terms and conditions of the Creative Commons Attribution license 3.0 Serbia (http://creativecommons.org/licenses/by/3.0/rs/). 